\title{
A Series of Lanthanide Coordination Polymers Based on Designed Bifunctional 1,4-Bis(imidazol-1-yl)terephthalic Acid Ligand: Structural Diversities, Luminescence, and Magnetic Properties
}

5 Xiu-Tang Zhang, ${ }^{* a, b}$ Li-Ming Fan, ${ }^{\text {a,b }}$ Wei-Liu Fan, ${ }^{\mathrm{b}}$ Bin Li, ${ }^{\mathrm{a}}$ Guang-Zeng Liu, ${ }^{\mathrm{a}}$ Xin-Zheng Liu, ${ }^{\mathrm{a}}$ and Xian Zhao* ${ }^{\mathrm{b}}$

${ }^{a}$ Advanced Material Institute of Research, College of Chemistry and Chemical Engineering, Qilu Normal University, Jinan, 250013, China.

${ }^{b}$ State Key Laboratory of Crystal Materials, Shandong University, Jinan 250100, China.

E-mail: zhaoxian@icm.sdu.edu.cn; xiutangzhang@163.com.

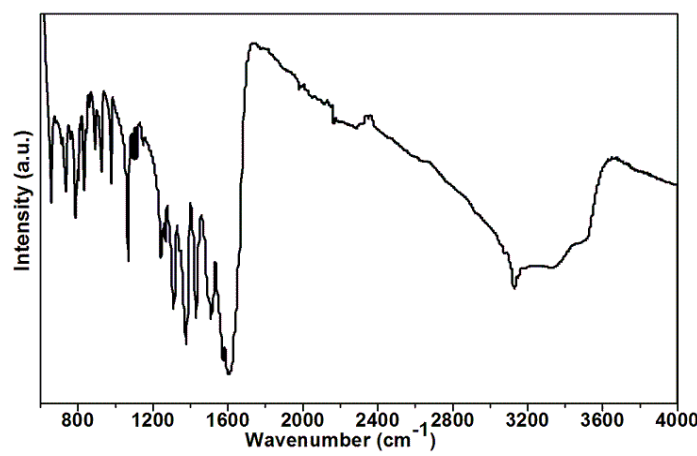

(a)

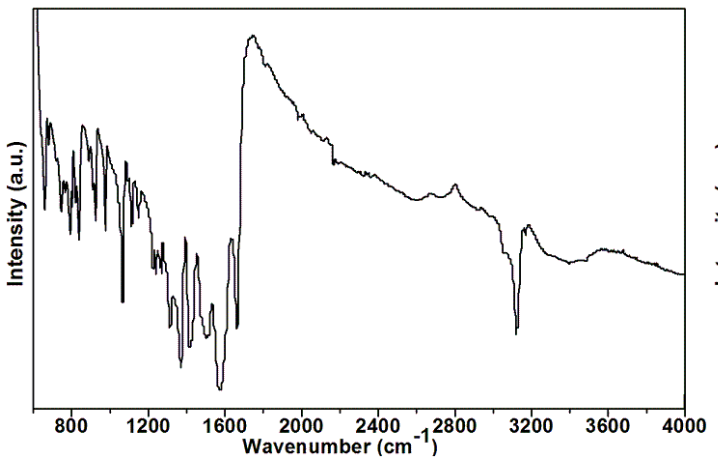

(c)

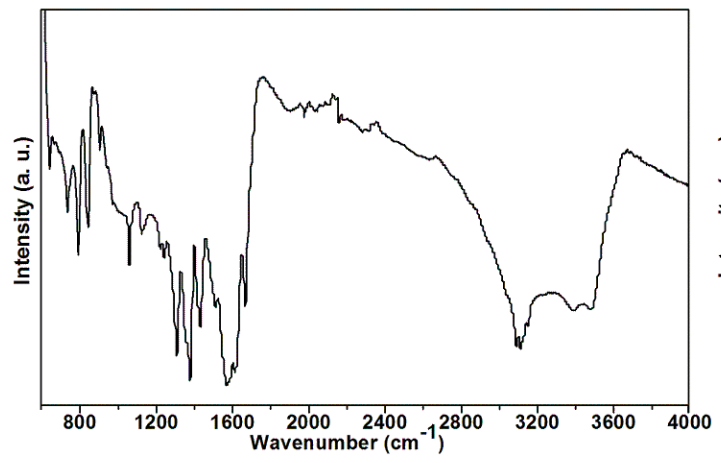

(e)

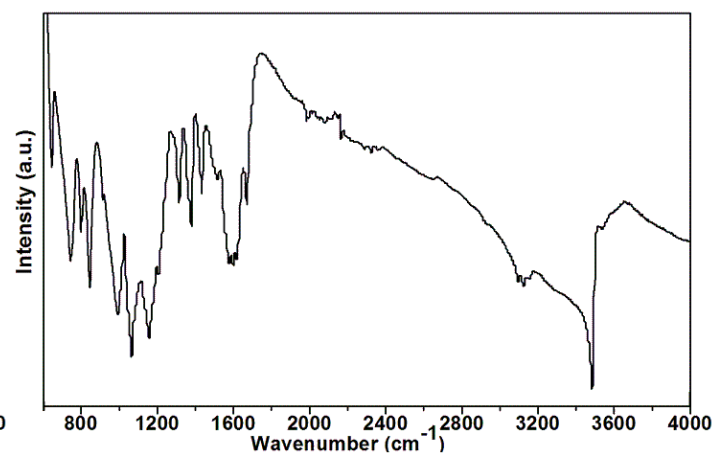

(b)

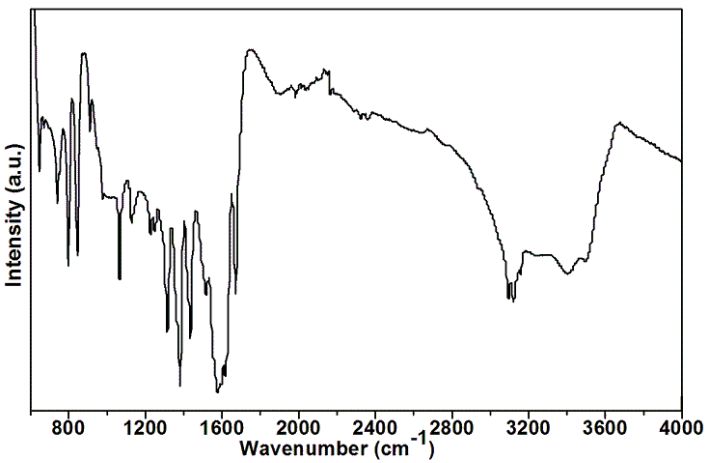

(d)

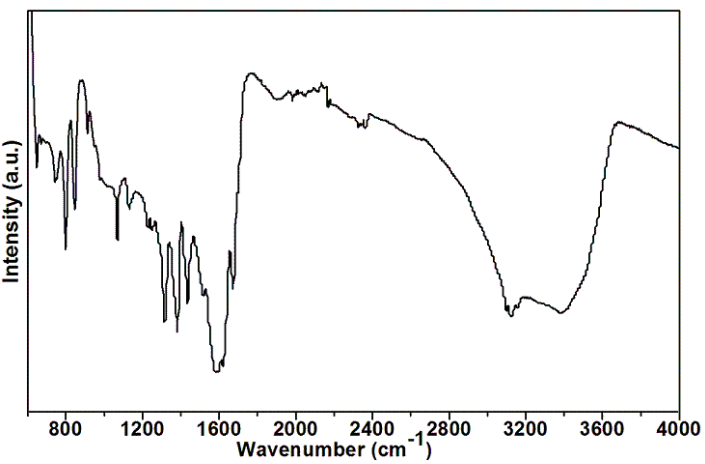

(f) 


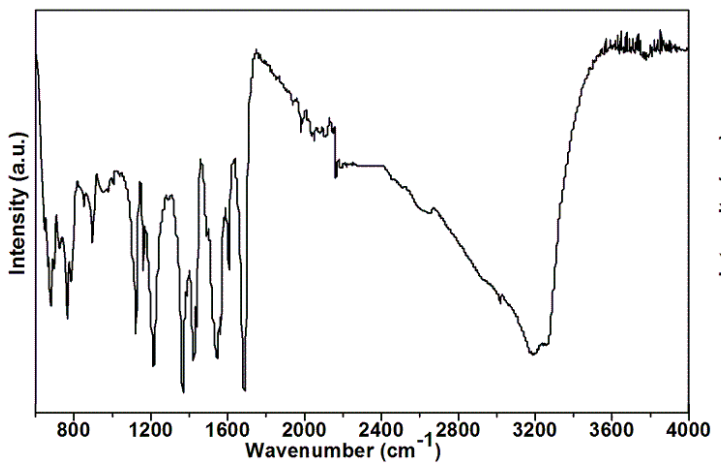

(e)

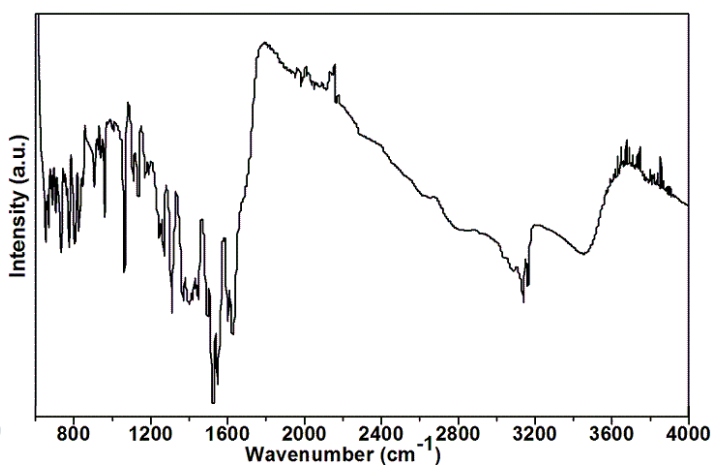

(f)

Fig. S1 The IR spectrum of complex 1-8 (a-h).

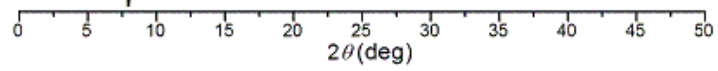

(a)

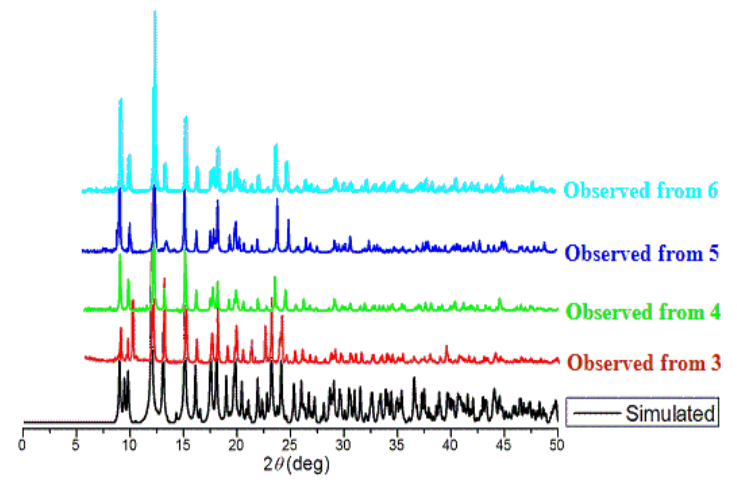

(c)

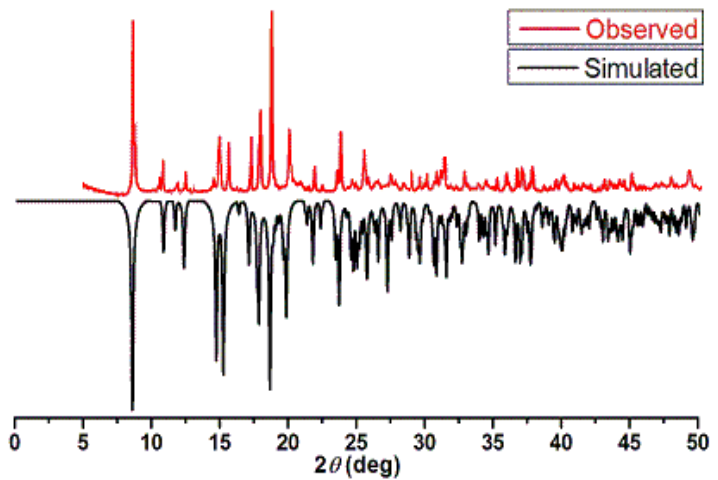

(b)

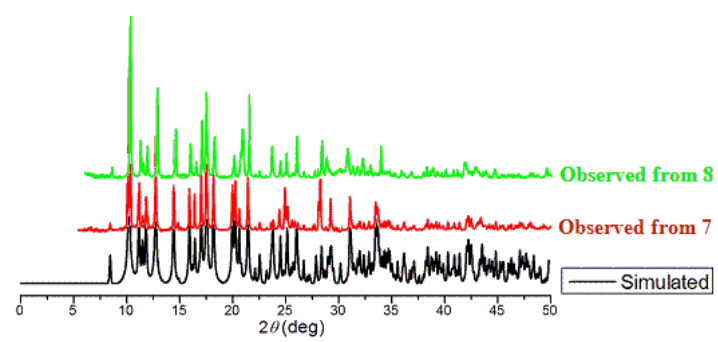

(d)

Fig. S2. PXRD patterns of 1 (a), 2 (b), 3-6 (c), and 7,8 (d). 


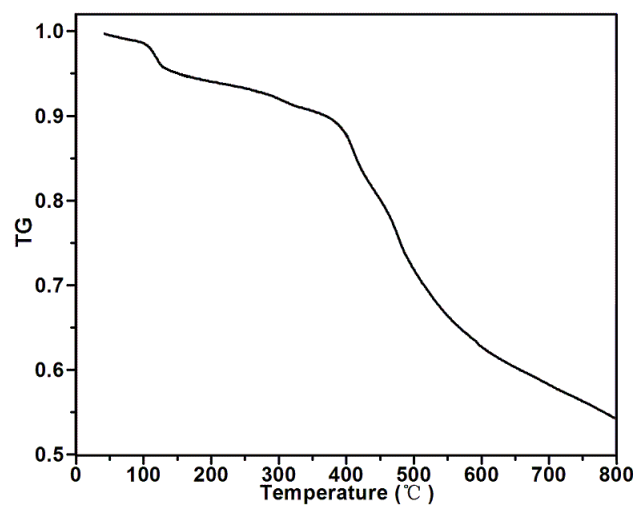

(a)

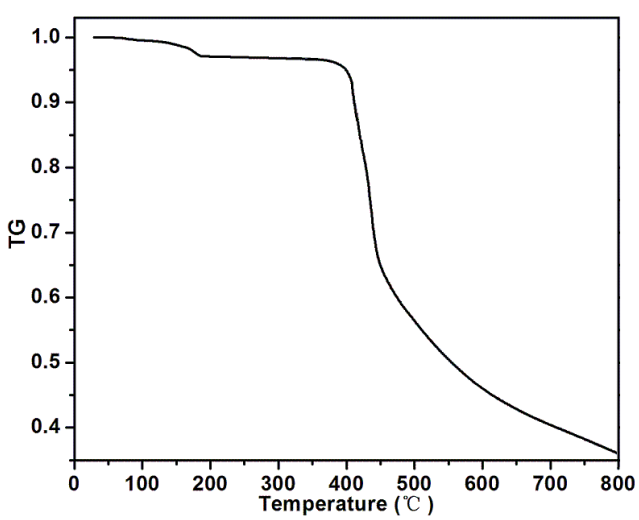

(c)

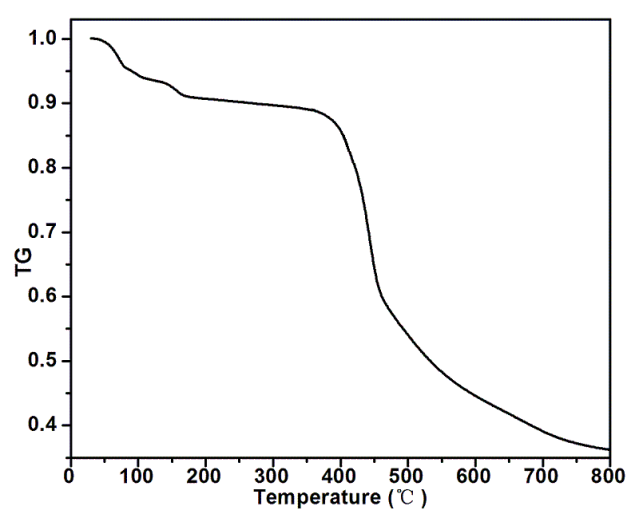

(e)

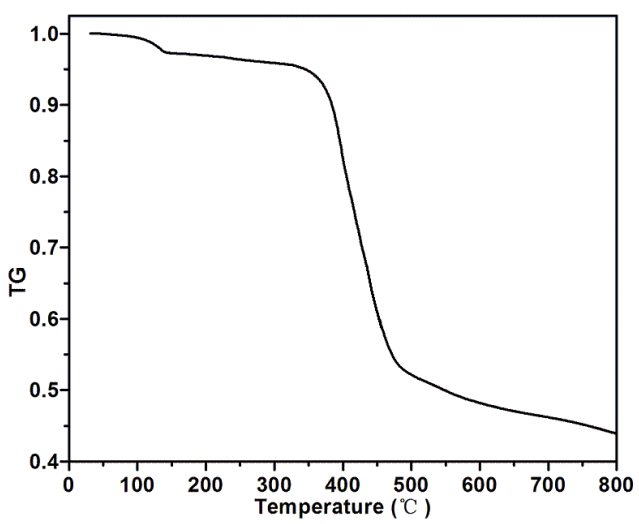

(g)

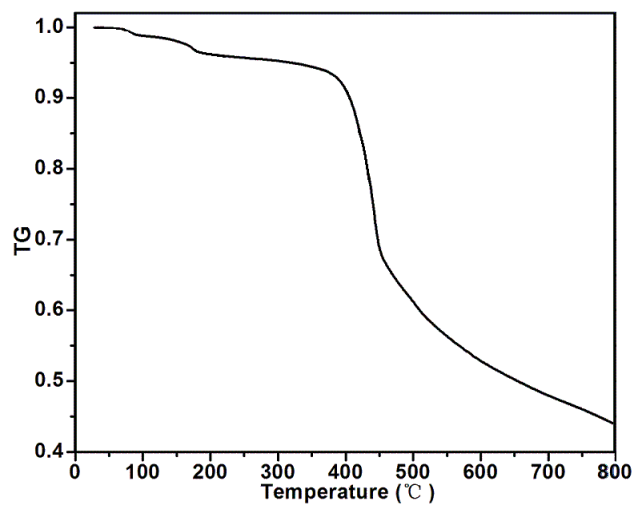

(b)

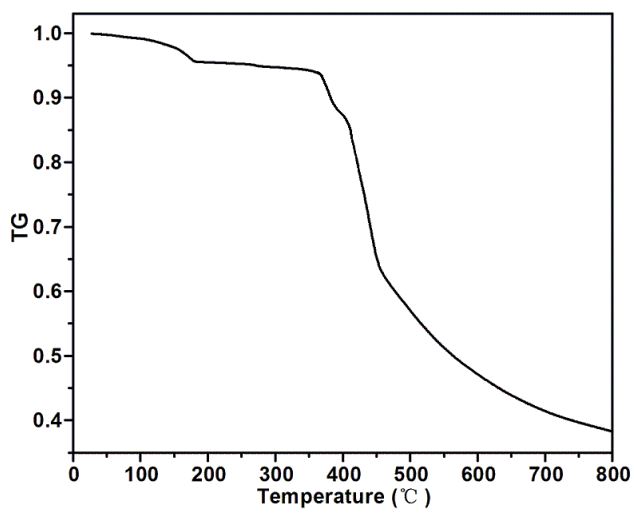

(d)

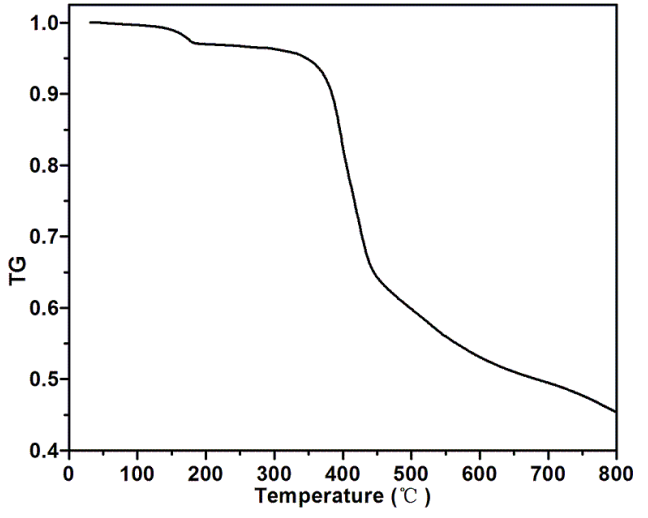

(f)

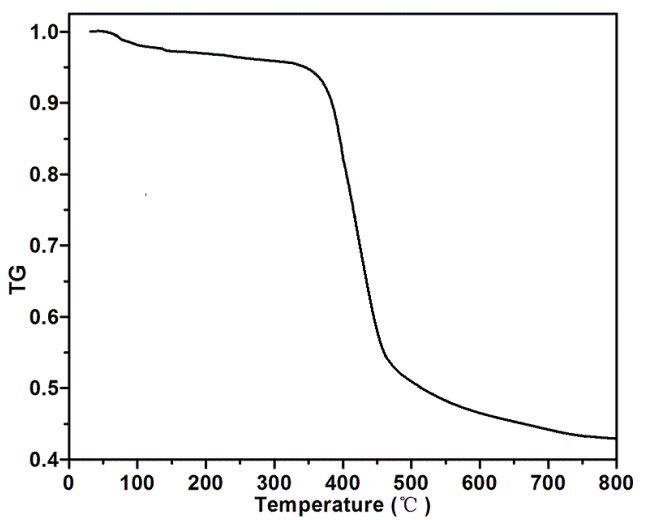

(h)

Fig. S3. TG curves of 1-8 (a-h). 


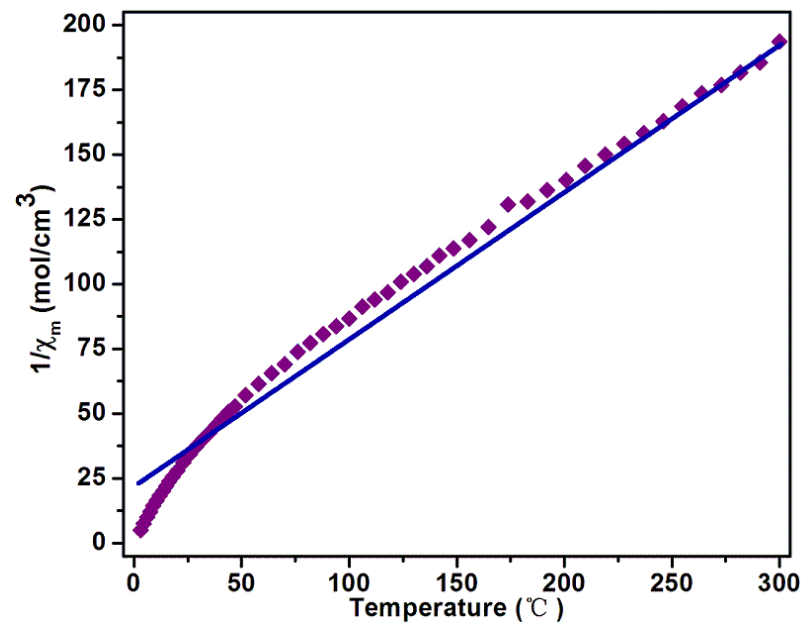

Fig S4. The $\chi_{m}{ }^{-1}-T$ curve of complex 2 (NdCP).

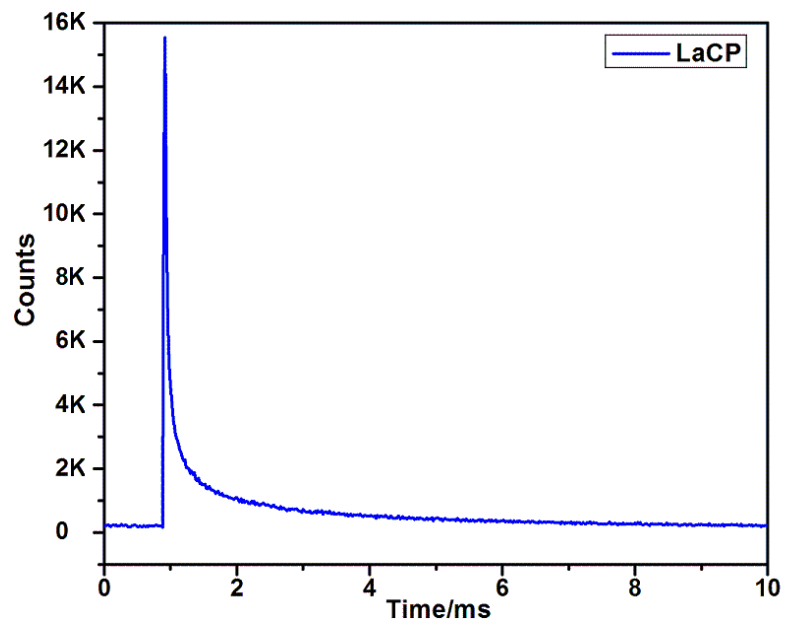

Fig S5. The decay profile of the complex 1 (LaCP).

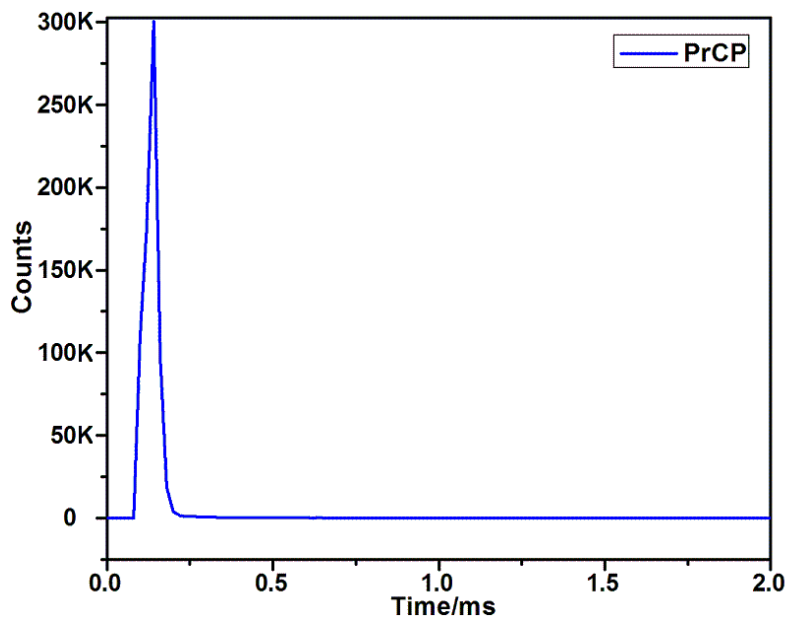

Fig S6. The decay profile of the complex 3 (PrCP). 


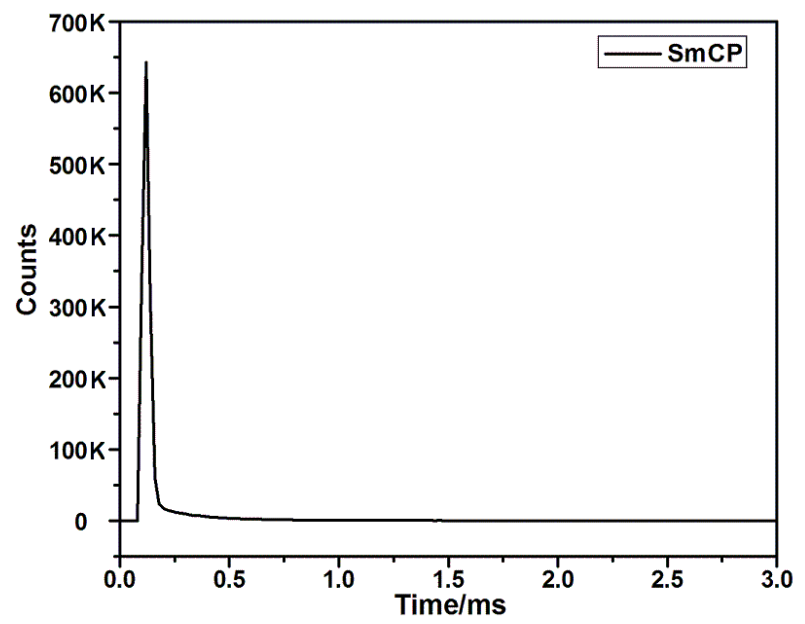

Fig S7. The decay profile of the complex 4 (SmCP).

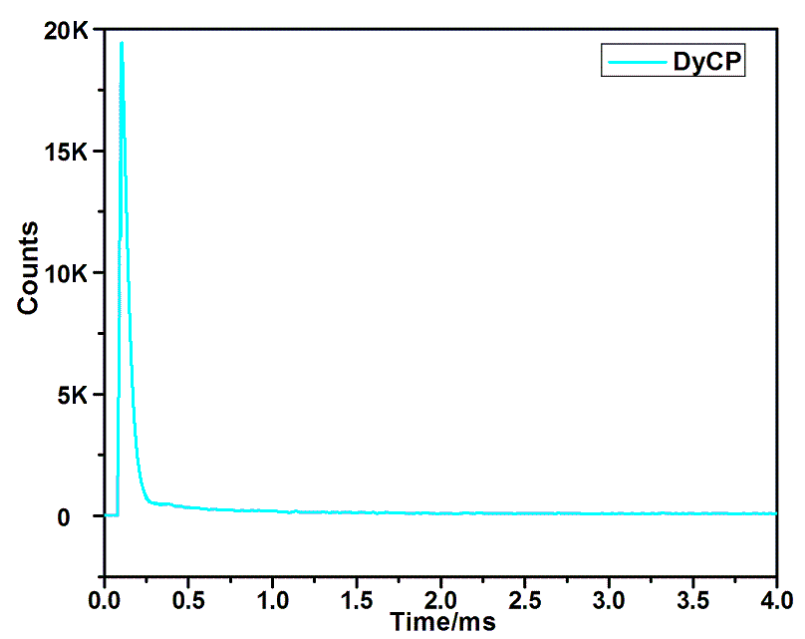

Fig S8. The decay profile of the complex 5 (DyCP).

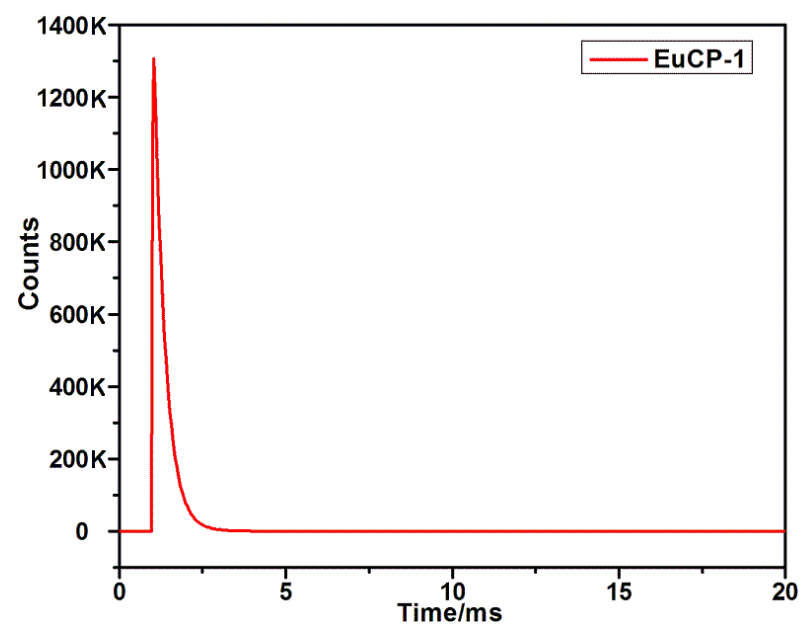

Fig S9. The decay profile of the complex 6 (EuCP-1). 


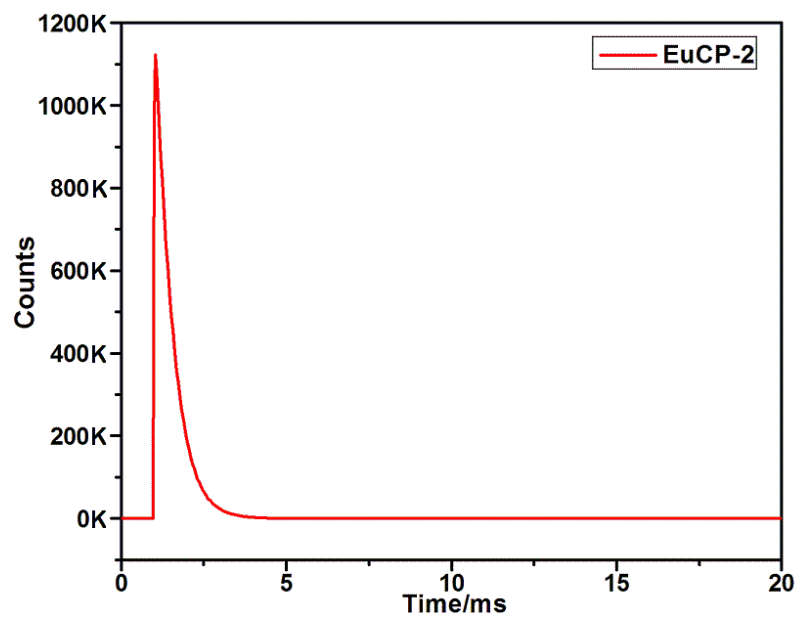

Fig S10. The decay profile of the complex 7 (EuCP-2).

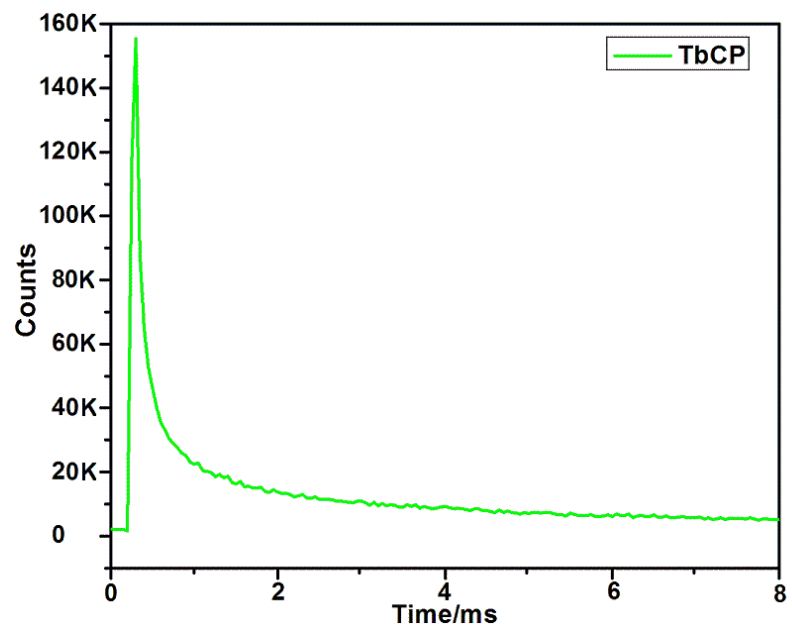

Fig S11. The decay profile of the complex 8 (TbCP). 
Table S1 Crystal data for $1-8$

\begin{tabular}{|c|c|c|c|c|c|c|c|c|}
\hline Complex & 1 & $\mathbf{2}$ & 3 & 4 & 5 & 6 & 7 & 8 \\
\hline $\begin{array}{l}\text { Empirical } \\
\text { formula }\end{array}$ & $\mathrm{C}_{24} \mathrm{H}_{18} \mathrm{La}_{2} \mathrm{~N}_{6} \mathrm{O}_{15}$ & $\mathrm{C}_{15} \mathrm{H}_{10} \mathrm{~N}_{4} \mathrm{NdO}_{7}$ & $\mathrm{C}_{16} \mathrm{H}_{11} \mathrm{~N}_{4} \mathrm{O}_{9} \mathrm{Pr}$ & $\mathrm{C}_{16} \mathrm{H}_{12} \mathrm{~N}_{4} \mathrm{O}_{9} \mathrm{Sm}$ & $\mathrm{C}_{16} \mathrm{H}_{9} \mathrm{DyN}_{4} \mathrm{O}_{9}$ & $\mathrm{C}_{16} \mathrm{H}_{8} \mathrm{EuN}_{4} \mathrm{O}_{9}$ & $\mathrm{C}_{28} \mathrm{H}_{20} \mathrm{Eu}_{2} \mathrm{~N}_{8} \mathrm{O}_{14} \mathrm{~S}$ & $\mathrm{C}_{28} \mathrm{H}_{20} \mathrm{~N}_{8} \mathrm{O}_{14} \mathrm{STb}_{2}$ \\
\hline $\begin{array}{l}\text { Formula } \\
\text { weight }\end{array}$ & 908.26 & 502.49 & 544.20 & 554.65 & 563.77 & 552.22 & 1028.50 & 1042.44 \\
\hline $\begin{array}{l}\text { Crystal } \\
\text { system }\end{array}$ & Triclinic & Monoclinic & Monoclinic & Monoclinic & Monoclinic & Monoclinic & Monoclinic & Monoclinic \\
\hline $\begin{array}{l}\text { Space } \\
\text { group }\end{array}$ & $P-1$ & $P 2_{1} / \mathrm{n}$ & $P 2_{1} / \mathrm{c}$ & $P 2_{1} / \mathrm{c}$ & $C 2 / \mathrm{m}$ & $C 2 / \mathrm{m}$ & $C 2 / \mathrm{c}$ & $C 2 / \mathrm{c}$ \\
\hline$a(\AA)$ & $11.0682(10)$ & $11.478(20)$ & $9.6646(16)$ & $10.436(3)$ & $10.3253(11)$ & $10.410(9)$ & $21.398(7)$ & $21.638(3)$ \\
\hline$b(\AA)$ & $11.3792(10)$ & $9.994(18)$ & $19.622(3)$ & $19.528(5)$ & $19.385(2)$ & $19.544(17)$ & $8.491(3)$ & $8.5417(12$ \\
\hline$c(\AA)$ & $11.6237(10)$ & $15.21(3)$ & $10.5041(17)$ & $9.507(3)$ & $9.3754(10)$ & $9.506(8)$ & $17.718(6)$ & $17.950(3)$ \\
\hline$\alpha\left({ }^{\circ}\right)$ & $80.8700(10)$ & 90.00 & 90.00 & 90.00 & 90.00 & 90.00 & 90.00 & 90.00 \\
\hline$\beta\left(^{\circ}\right)$ & $89.7280(10)$ & $108.46(3)$ & $104.716(2)$ & $103.875(4)$ & $103.035(2)$ & $103.677(12)$ & $104.783(4)$ & $105.085(2)$ \\
\hline$\gamma\left({ }^{\circ}\right)$ & $74.1040(10)$ & 90.00 & 90.00 & 90.00 & 90.00 & 90.00 & 90.00 & 90.00 \\
\hline$V\left(\AA^{3}\right)$ & $1389.0(2)$ & $1656(5)$ & $1926.6(6)$ & $1880.9(9)$ & $1828.2(3)$ & 1879(3) & $3112.7(17)$ & $3203.3(8)$ \\
\hline Z & 2 & 4 & 4 & 4 & 4 & 4 & 4 & 4 \\
\hline $\begin{array}{l}D_{\text {calcd }} \\
\left(\mathrm{Mg} / \mathrm{m}^{3}\right)\end{array}$ & 2.172 & 2.009 & 1.876 & 1.959 & 2.048 & 1.952 & 2.195 & 2.162 \\
\hline$\mu\left(\mathrm{mm}^{-1}\right)$ & 3.123 & 3.185 & 2.587 & 3.182 & 4.148 & 3.397 & 4.148 & 4.530 \\
\hline$T(\mathrm{~K})$ & $293(2)$ & $296(2)$ & $296(2)$ & 293(2) & $293(2)$ & $298(2)$ & $296(2)$ & 293(2) \\
\hline$R_{\text {int }}$ & 0.0136 & 0.0288 & 0.0166 & 0.0439 & 0.0223 & 0.3357 & 0.0198 & 0.0186 \\
\hline$R_{1}\left(w R_{2}\right)[I$ & 0.0190 & 0.1360 & 0.0174 & 0.0458 & 0.0176 & 0.0845 & 0.0205 & 0.0224 \\
\hline$>2 \sigma(I)]^{\mathrm{a}^{2}}$ & 0.0629 & 0.3932 & 0.0491 & 0.1214 & 0.0492 & 0.1909 & 0.0550 & 0.0615 \\
\hline$R_{1} \quad\left(w R_{2}\right)$ & 0.0201 & 0.1762 & 0.0189 & 0.0475 & 0.0185 & 0.0995 & 0.0219 & 0.0242 \\
\hline$(\text { all data })^{\mathrm{a}}$ & 0.0635 & 0.4819 & 0.0501 & 0.1235 & 0.0498 & 0.2154 & 0.0557 & 0.0732 \\
\hline Gof & 1.038 & 2.233 & 1.083 & 1.093 & 1.016 & 1.072 & 1.101 & 1.181 \\
\hline \multicolumn{9}{|c|}{$\left.{ }^{a} R_{1}=\Sigma|| F_{\mathrm{o}}|-| F_{\mathrm{c}}|| / \Sigma\left|F_{\mathrm{o}}\right|, w R_{2}=\left[\Sigma w\left(F_{\mathrm{o}}^{2}-F_{\mathrm{c}}^{2}\right)^{2}\right] / \Sigma w\left(F_{\mathrm{o}}^{2}\right)^{2}\right]^{1 / 2}$} \\
\hline
\end{tabular}


Table S2 Selected bond lengths $(\AA)$ and angles $\left({ }^{\circ}\right)$ for $\mathbf{1}-\mathbf{6}$

\begin{tabular}{|c|c|c|c|c|c|c|c|}
\hline Complex 1 & & & & & & & \\
\hline $\mathrm{La}(1)-\mathrm{O}(2)$ & $2.435(3)$ & $\mathrm{La}(1)-\mathrm{O}(10)^{\# 3}$ & $2.582(3)$ & $\mathrm{La}(2)-\mathrm{O}(14)$ & $2.477(3)$ & $\mathrm{La}(2)-\mathrm{O}(11)$ & $2.666(3)$ \\
\hline $\mathrm{La}(1)-\mathrm{O}(6)^{\# 1}$ & $2.538(3)$ & $\mathrm{La}(1)-\mathrm{O}(4)^{\# 2}$ & $2.634(3)$ & $\mathrm{La}(2)-\mathrm{O}(9)$ & $2.509(3)$ & $\mathrm{La}(2)-\mathrm{N}(4)$ & $2.666(4)$ \\
\hline $\mathrm{La}(1)-\mathrm{O}(5)$ & $2.538(3)$ & $\mathrm{La}(1)-\mathrm{N}(5)^{\# 4}$ & $2.727(4)$ & $\mathrm{La}(2)-\mathrm{O}(1)^{\# 5}$ & $2.537(3)$ & $\mathrm{La}(2)-\mathrm{O}(8)^{\# 5}$ & $2.689(3)$ \\
\hline $\mathrm{La}(1)-\mathrm{O}(3)^{\# 2}$ & $2.565(3)$ & $\mathrm{La}(1)-\mathrm{O}(10)^{\# 5}$ & $2.743(3)$ & $\mathrm{La}(2)-\mathrm{O}(15)$ & $2.571(3)$ & $\mathrm{La}(2)-\mathrm{O}(8)$ & $2.703(3)$ \\
\hline $\mathrm{La}(1)-\mathrm{O}(7)^{\# 3}$ & $2.582(3)$ & $\mathrm{La}(2)-\mathrm{O}(12)$ & $2.449(3)$ & $\mathrm{O}(1)^{\# 5}-\mathrm{La}(2)-\mathrm{N}(4)$ & $139.21(12)$ & $\mathrm{O}(14)-\mathrm{La}(2)-\mathrm{O}(11)$ & $71.28(11)$ \\
\hline $\mathrm{O}(2)-\mathrm{La}(1)-\mathrm{O}(6)^{\# 1}$ & $75.78(10)$ & $\mathrm{O}(3)^{\# 2}-\mathrm{La}(1)-\mathrm{O}(4)^{\# 2}$ & $50.23(10)$ & $\mathrm{O}(12)-\mathrm{La}(2)-\mathrm{O}(9)$ & $144.08(11)$ & $\mathrm{O}(12)-\mathrm{La}(2)-\mathrm{O}(14)$ & $74.14(11)$ \\
\hline $\mathrm{O}(2)-\mathrm{La}(1)-\mathrm{O}(5)$ & $132.84(10)$ & $\mathrm{O}(7)^{\# 3}-\mathrm{La}(1)-\mathrm{O}(4)^{\# 2}$ & $71.66(10)$ & $\mathrm{O}(14)-\mathrm{La}(2)-\mathrm{O}(9)$ & $132.13(10)$ & $\mathrm{O}(11)-\mathrm{La}(2)-\mathrm{N}(4)$ & $70.61(12)$ \\
\hline $\mathrm{O}(6)^{\# 1}-\mathrm{La}(1)-\mathrm{O}(5)$ & $64.03(9)$ & $\mathrm{O}(10)^{\# 3}-\mathrm{La}(1)-\mathrm{O}(4)^{\# 2}$ & $96.26(10)$ & $\mathrm{O}(3)^{\# 2}-\mathrm{La}(1)-\mathrm{O}(10)^{\# 3}$ & $135.74(10)$ & $\mathrm{O}(12)-\mathrm{La}(2)-\mathrm{O}(8)^{\# 5}$ & $71.05(10)$ \\
\hline $\mathrm{O}(2)-\mathrm{La}(1)-\mathrm{O}(3)^{\# 2}$ & $116.34(10)$ & $\mathrm{O}(2)-\mathrm{La}(1)-\mathrm{N}(5)^{\nexists 4}$ & $71.12(11)$ & $\mathrm{O}(14)-\mathrm{La}(2)-\mathrm{O}(1)^{\# 5}$ & $143.26(11)$ & $\mathrm{O}(14)-\mathrm{La}(2)-\mathrm{O}(8)^{\# 5}$ & $87.03(10)$ \\
\hline $\mathrm{O}(6)^{\# 1}-\mathrm{La}(1)-\mathrm{O}(3)^{\# 2}$ & $70.88(10)$ & $\mathrm{O}(6)^{\# 1}-\mathrm{La}(1)-\mathrm{N}(5)^{\# 4}$ & $73.06(11)$ & $\mathrm{O}(15)-\mathrm{La}(2)-\mathrm{N}(4)$ & $137.83(13)$ & $\mathrm{O}(9)-\mathrm{La}(2)-\mathrm{O}(8)^{\# 5}$ & $125.47(9)$ \\
\hline $\mathrm{O}(5)-\mathrm{La}(1)-\mathrm{O}(3)^{\# 2}$ & $73.46(10)$ & $\mathrm{O}(5)-\mathrm{La}(1)-\mathrm{N}(5)^{\# 4}$ & $74.29(11)$ & $\mathrm{O}(12)-\mathrm{La}(2)-\mathrm{O}(15)$ & $70.95(12)$ & $\mathrm{O}(1)^{\# 5}-\mathrm{La}(2)-\mathrm{O}(8)^{\# 5}$ & $98.72(9)$ \\
\hline $\mathrm{O}(2)-\mathrm{La}(1)-\mathrm{O}(7)^{\# 3}$ & $126.02(10)$ & $\mathrm{O}(3)^{\# 2}-\mathrm{La}(1)-\mathrm{N}(5)^{\# 4}$ & $139.32(11)$ & $\mathrm{O}(15)-\mathrm{La}(2)-\mathrm{O}(8)^{\# 5}$ & $141.89(10)$ & $\mathrm{O}(9)-\mathrm{La}(2)-\mathrm{O}(1)^{* 5}$ & $72.37(10)$ \\
\hline $\mathrm{O}(6)^{\# 1}-\mathrm{La}(1)-\mathrm{O}(7)^{\# 3}$ & $129.18(9)$ & $\mathrm{O}(7)^{\# 3}-\mathrm{La}(1)-\mathrm{N}(5)^{\# 4}$ & $73.55(11)$ & $\mathrm{O}(11)-\mathrm{La}(2)-\mathrm{O}(8)^{\# 5}$ & $139.58(10)$ & $\mathrm{O}(14)-\mathrm{La}(2)-\mathrm{O}(15)$ & $80.08(13)$ \\
\hline $\mathrm{O}(5)-\mathrm{La}(1)-\mathrm{O}(7)^{+3}$ & $70.74(9)$ & $\mathrm{O}(10)^{\# 3}-\mathrm{La}(1)-\mathrm{N}(5)^{\# 4}$ & $84.75(11)$ & $\mathrm{O}(1)^{75}-\mathrm{La}(2)-\mathrm{O}(15)$ & $73.13(12)$ & $\mathrm{N}(4)-\mathrm{La}(2)-\mathrm{O}(8)^{* 5}$ & $71.51(10)$ \\
\hline $\mathrm{O}(3)^{\# 2}-\mathrm{La}(1)-\mathrm{O}(7)^{\# 3}$ & $117.16(10)$ & $\mathrm{O}(4)^{\# 2}-\mathrm{La}(1)-\mathrm{N}(5)^{\# 4}$ & $139.76(11)$ & $\mathrm{O}(12)-\mathrm{La}(2)-\mathrm{O}(11)$ & $130.16(11)$ & $\mathrm{O}(12)-\mathrm{La}(2)-\mathrm{O}(1)^{\# 5}$ & $73.63(10)$ \\
\hline $\mathrm{O}(2)-\mathrm{La}(1)-\mathrm{O}(10)^{\# 3}$ & $75.13(10)$ & $\mathrm{O}(2)-\mathrm{La}(1)-\mathrm{O}(10)^{\# 5}$ & $70.79(10)$ & $\mathrm{O}(14)-\mathrm{La}(2)-\mathrm{O}(11)$ & $71.28(11)$ & $\mathrm{O}(9)-\mathrm{La}(2)-\mathrm{O}(15)$ & $88.32(11)$ \\
\hline $\mathrm{O}(11)-\mathrm{La}(2)-\mathrm{O}(8)$ & $115.01(10)$ & $\mathrm{O}(6)^{\# 1}-\mathrm{La}(1)-\mathrm{O}(10)^{\# 5}$ & $119.69(9)$ & $\mathrm{O}(12)-\mathrm{La}(2)-\mathrm{O}(8)$ & $114.33(10)$ & $\mathrm{O}(9)-\mathrm{La}(2)-\mathrm{O}(8)$ & $62.47(9)$ \\
\hline $\mathrm{O}(5)-\mathrm{La}(1)-\mathrm{O}(10)^{\# 3}$ & $132.13(9)$ & $\mathrm{O}(5)-\mathrm{La}(1)-\mathrm{O}(10)^{\# 5}$ & $151.29(9)$ & $\mathrm{O}(1)^{\# 5}-\mathrm{La}(2)-\mathrm{O}(11)$ & $119.21(11)$ & $\mathrm{O}(1)^{+5}-\mathrm{La}(2)-\mathrm{O}(8)$ & $68.70(10)$ \\
\hline $\mathrm{O}(14)-\mathrm{La}(2)-\mathrm{N}(4)$ & $77.07(12)$ & $\mathrm{O}(3)^{\# 2}-\mathrm{La}(1)-\mathrm{O}(10)^{\# 5}$ & $81.09(10)$ & $\mathrm{O}(6)^{\# 1}-\mathrm{La}(1)-\mathrm{O}(10)^{\# 3}$ & $147.82(10)$ & $\mathrm{O}(15)-\mathrm{La}(2)-\mathrm{O}(11)$ & $68.65(13)$ \\
\hline $\mathrm{O}(12)-\mathrm{La}(2)-\mathrm{N}(4)$ & $133.32(11)$ & $\mathrm{O}(7)^{\# 3}-\mathrm{La}(1)-\mathrm{O}(10)^{\# 5}$ & $111.07(9)$ & $\mathrm{O}(15)-\mathrm{La}(2)-\mathrm{O}(8)$ & $137.23(11)$ & $\mathrm{O}(9)-\mathrm{La}(2)-\mathrm{O}(11)$ & $61.28(10)$ \\
\hline $\mathrm{O}(2)-\mathrm{La}(1)-\mathrm{O}(4)^{\# 2}$ & $148.01(10)$ & $\mathrm{O}(10)^{\# 3}-\mathrm{La}(1)-\mathrm{O}(10)^{\# 5}$ & $61.85(11)$ & $\mathrm{O}(7)^{\# 3}-\mathrm{La}(1)-\mathrm{O}(10)^{\# 3}$ & $62.11(9)$ & $\mathrm{N}(4)-\mathrm{La}(2)-\mathrm{O}(8)$ & $71.57(11)$ \\
\hline $\mathrm{O}(6)^{\# 1}-\mathrm{La}(1)-\mathrm{O}(4)^{\# 2}$ & $115.76(9)$ & $\mathrm{O}(4)^{\# 2}-\mathrm{La}(1)-\mathrm{O}(10)^{\# 5}$ & $77.95(10)$ & $\mathrm{O}(14)-\mathrm{La}(2)-\mathrm{O}(8)$ & $142.65(11)$ & $\mathrm{O}(8)^{\# 5}-\mathrm{La}(2)-\mathrm{O}(8)$ & $64.28(10)$ \\
\hline $\mathrm{O}(5)-\mathrm{La}(1)-\mathrm{O}(4)^{\# 2}$ & $75.64(10)$ & $\mathrm{N}(5)^{\# 4}-\mathrm{La}(1)-\mathrm{O}(10)^{\# 5}$ & $134.35(10)$ & & & & \\
\hline Symmetry codes: $\# 1$ - & $x-1-2 \cdot \# 2 x$ & $1-\# 2 x-1 \cdot+4$ & $-75-1$ & & & & \\
\hline Complex 2 & & & & & & & \\
\hline $\mathrm{Nd}(1)-\mathrm{O}(4)$ & $2.431(11)$ & $\mathrm{Nd}(1)-\mathrm{O}(6)^{\# 5}$ & $2.457(13)$ & $\mathrm{Nd}(1)-\mathrm{O}(1)$ & $2.547(17)$ & $\mathrm{Nd}(1)-\mathrm{N}(1)^{\# 6}$ & $2.69(2)$ \\
\hline $\mathrm{Nd}(1)-\mathrm{O}(5)^{\# 4}$ & $2.447(12)$ & $\mathrm{Nd}(1)-\mathrm{O}(3)$ & $2.524(11)$ & $\mathrm{Nd}(1)-\mathrm{O}(2)^{\# 1}$ & $2.570(11)$ & $\mathrm{Nd}(1)-\mathrm{N}(4)^{\# 7}$ & $2.696(16)$ \\
\hline $\mathrm{O}(4)-\mathrm{Nd}(1)-\mathrm{O}(5)^{\# 4}$ & $82.6(4)$ & $\mathrm{O}(5)^{\# 4}-\mathrm{Nd}(1)-\mathrm{O}(1)$ & $142.6(4)$ & $\mathrm{O}(1)-\mathrm{Nd}(1)-\mathrm{O}(2)^{\# 1}$ & $65.0(4)$ & $\mathrm{O}(4)-\mathrm{Nd}(1)-\mathrm{N}(4)^{\# 7}$ & $85.7(4)$ \\
\hline $\mathrm{O}(4)-\mathrm{Nd}(1)-\mathrm{O}(6)^{\# 5}$ & $148.8(5)$ & $\mathrm{O}(6)^{\# 5}-\mathrm{Nd}(1)-\mathrm{O}(1)$ & $84.1(4)$ & $\mathrm{O}(4)-\mathrm{Nd}(1)-\mathrm{N}(1)^{\# 6}$ & $122.2(5)$ & $\mathrm{O}(5)^{\# 4}-\mathrm{Nd}(1)-\mathrm{N}(4)^{\# 7}$ & $143.9(4)$ \\
\hline $\mathrm{O}(5)^{\# 4}-\mathrm{Nd}(1)-\mathrm{O}(6)^{\# 5}$ & $81.6(5)$ & $\mathrm{O}(3)-\mathrm{Nd}(1)-\mathrm{O}(1)$ & $134.7(4)$ & $\mathrm{O}(5)^{\# 4}-\mathrm{Nd}(1)-\mathrm{N}(1)^{\# 6}$ & $71.8(5)$ & $\mathrm{O}(6)^{\# 5}-\mathrm{Nd}(1)-\mathrm{N}(4)^{\# 7}$ & $91.5(5)$ \\
\hline $\mathrm{O}(4)-\mathrm{Nd}(1)-\mathrm{O}(3)$ & $72.4(4)$ & $\mathrm{O}(4)-\mathrm{Nd}(1)-\mathrm{O}(2)^{\# 1}$ & $70.1(4)$ & $\mathrm{O}(6)^{\# 5}-\mathrm{Nd}(1)-\mathrm{N}(1)^{\# 6}$ & $77.4(6)$ & $\mathrm{O}(3)-\mathrm{Nd}(1)-\mathrm{N}(4)^{\# 7}$ & 68.9(4) \\
\hline $\mathrm{O}(5)^{\# 4}-\mathrm{Nd}(1)-\mathrm{O}(3)$ & $75.0(5)$ & $\mathrm{O}(5)^{\# 4}-\mathrm{Nd}(1)-\mathrm{O}(2)^{\# 1}$ & $108.0(5)$ & $\mathrm{O}(3)-\mathrm{Nd}(1)-\mathrm{N}(1)^{\# 6}$ & $140.8(5)$ & $\mathrm{O}(1)-\mathrm{Nd}(1)-\mathrm{N}(4)^{\# 7}$ & $70.6(4)$ \\
\hline $\mathrm{O}(6)^{\# 5}-\mathrm{Nd}(1)-\mathrm{O}(3)$ & $77.7(4)$ & $\mathrm{O}(6)^{\# 5}-\mathrm{Nd}(1)-\mathrm{O}(2)^{\# 1}$ & $140.7(4)$ & $\mathrm{O}(1)-\mathrm{Nd}(1)-\mathrm{N}(1)^{\# 6}$ & $71.4(5)$ & $\mathrm{O}(2)^{\# 1}-\mathrm{Nd}(1)-\mathrm{N}(4)^{\# 7}$ & $99.7(4)$ \\
\hline $\mathrm{O}(4)-\mathrm{Nd}(1)-\mathrm{O}(1)$ & $123.5(4)$ & $\mathrm{O}(3)-\mathrm{Nd}(1)-\mathrm{O}(2)^{\# 1}$ & $141.4(4)$ & $\mathrm{O}(2)^{\# 1}-\mathrm{Nd}(1)-\mathrm{N}(1)^{\# 6}$ & $70.3(5)$ & $\mathrm{N}(1)^{\# 6}-\mathrm{Nd}(1)-\mathrm{N}(4)^{\# 7}$ & $141.2(6)$ \\
\hline Symmetry codes: $\# 1-3$ & $-x+2,-y+2,-z$ & $4-x+2,-y+1,-z ; \# 5 x+1, y$, & $z ; \# 6 x+1 / 2,-y$ & $3 / 2, z-1 / 2 ; \# 7 x+1 / 2,-y+$ & $3 / 2, z+1 / 2$ & & \\
\hline Complex 3 & & & & & & & \\
\hline $\mathrm{O}(1)-\operatorname{Pr}(1)$ & $2.5300(17)$ & $\mathrm{O}(6)-\operatorname{Pr}(1)$ & $2.4892(16)$ & $\mathrm{O}(8)-\operatorname{Pr}(1)$ & $2.5086(19)$ & $\operatorname{Pr}(1)-\mathrm{O}(3)^{\# 4}$ & $2.5022(17)$ \\
\hline $\mathrm{O}(2)-\operatorname{Pr}(1)$ & $2.5971(17)$ & $\mathrm{O}(7)-\operatorname{Pr}(1)$ & $2.5190(18)$ & $\mathrm{O}(9)-\operatorname{Pr}(1)$ & $2.4869(19)$ & $\operatorname{Pr}(1)-\mathrm{O}(4)^{\# 4}$ & $2.6220(17)$ \\
\hline $\mathrm{O}(5)-\operatorname{Pr}(1)$ & $2.4691(17)$ & $\mathrm{O}(3)^{\# 4}-\operatorname{Pr}(1)-\mathrm{O}(8)$ & $96.42(6)$ & $\mathrm{O}(3)^{\# 4}-\operatorname{Pr}(1)-\mathrm{O}(1)$ & 127.2 & $\mathrm{O}(1)-\operatorname{Pr}(1)-\mathrm{O}(2)$ & 7(5) \\
\hline $\mathrm{O}(5)-\operatorname{Pr}(1)-\mathrm{O}(9)$ & $83.18(6)$ & $\mathrm{O}(5)-\operatorname{Pr}(1)-\mathrm{O}(7)$ & $80.98(7)$ & $\mathrm{O}(8)-\operatorname{Pr}(1)-\mathrm{O}(1)$ & $74.38(6)$ & $\mathrm{O}(5)-\operatorname{Pr}(1)-\mathrm{O}(4)^{\# 4}$ & $72.91(6)$ \\
\hline $\mathrm{O}(5)-\operatorname{Pr}(1)-\mathrm{O}(6)$ & $66.10(6)$ & $O(9)-\operatorname{Pr}(1)-O(7)$ & $142.93(6)$ & $\mathrm{O}(7)-\operatorname{Pr}(1)-\mathrm{O}(1)$ & $81.27(7)$ & $\mathrm{O}(9)-\operatorname{Pr}(1)-\mathrm{O}(4)^{\# 4}$ & $81.38(5)$ \\
\hline $\mathrm{O}(9)-\operatorname{Pr}(1)-\mathrm{O}(6)$ & $72.43(6)$ & $\mathrm{O}(6)-\operatorname{Pr}(1)-\mathrm{O}(7)$ & $70.52(6)$ & $\mathrm{O}(5)-\operatorname{Pr}(1)-\mathrm{O}(2)$ & $146.49(6)$ & $\mathrm{O}(6)-\operatorname{Pr}(1)-\mathrm{O}(4)^{\# 4}$ & $133.18(6)$ \\
\hline $\mathrm{O}(5)-\operatorname{Pr}(1)-\mathrm{O}(3)^{\# 4}$ & $80.03(6)$ & $\mathrm{O}(3)^{\# 4}-\operatorname{Pr}(1)-\mathrm{O}(7)$ & $77.42(6)$ & $\mathrm{O}(9)-\operatorname{Pr}(1)-\mathrm{O}(2)$ & $130.33(6)$ & $\mathrm{O}(7)-\operatorname{Pr}(1)-\mathrm{O}(2)$ & $71.21(6)$ \\
\hline $\mathrm{O}(9)-\operatorname{Pr}(1)-\mathrm{O}(3)^{\# 4}$ & $132.08(5)$ & $\mathrm{O}(8)-\operatorname{Pr}(1)-\mathrm{O}(7)$ & $143.85(6)$ & $\mathrm{O}(6)-\operatorname{Pr}(1)-\mathrm{O}(2)$ & $118.75(6)$ & $\mathrm{O}(8)-\operatorname{Pr}(1)-\mathrm{O}(4)^{\# 4}$ & $69.75(5)$ \\
\hline $\mathrm{O}(6)-\mathrm{Pr}(1)-\mathrm{O}(3)^{\# 4}$ & $136.03(6)$ & $\mathrm{O}(5)-\operatorname{Pr}(1)-\mathrm{O}(1)$ & $142.57(6)$ & $\mathrm{O}(3)^{\# 4}-\operatorname{Pr}(1)-\mathrm{O}(2)$ & $76.03(6)$ & $\mathrm{O}(7)-\operatorname{Pr}(1)-\mathrm{O}(4)^{\# 4}$ & $124.62(6)$ \\
\hline $\mathrm{O}(5)-\operatorname{Pr}(1)-\mathrm{O}(8)$ & $133.64(6)$ & $\mathrm{O}(9)-\operatorname{Pr}(1)-\mathrm{O}(1)$ & $91.65(6)$ & $\mathrm{O}(8)-\operatorname{Pr}(1)-\mathrm{O}(2)$ & $72.73(6)$ & $\mathrm{O}(1)-\operatorname{Pr}(1)-\mathrm{O}(4)^{\# 4}$ & $143.03(7)$ \\
\hline $\mathrm{O}(9)-\operatorname{Pr}(1)-\mathrm{O}(8)$ & $65.09(6)$ & $\mathrm{O}(6)-\operatorname{Pr}(1)-\mathrm{O}(1)$ & $76.97(6)$ & $\mathrm{O}(3)^{\# 4}-\operatorname{Pr}(1)-\mathrm{O}(4)^{\# 4}$ & $50.81(5)$ & $\mathrm{O}(2)-\operatorname{Pr}(1)-\mathrm{O}(4)^{\# 4}$ & $107.86(6)$ \\
\hline $\mathrm{O}(6)-\operatorname{Pr}(1)-\mathrm{O}(8)$ & $127.12(6)$ & & & & & & \\
\hline Symmetry code: $\# 4-x$ & re+1/0 & & & & & & \\
\hline & & & & & & & \\
\hline $\mathrm{Sm}(1)-\mathrm{O}(6)$ & $2.430(7)$ & $\operatorname{Sm}(1)-\mathrm{O}(2)^{\# 2}$ & $2.480(9)$ & $\mathrm{Sm}(1)-\mathrm{O}(3)$ & $2.519(7)$ & $\mathrm{Sm}(1)-\mathrm{O}(4 \mathrm{~A})$ & $2.573(13)$ \\
\hline $\mathrm{Sm}(1)-\mathrm{O}(1)$ & $2.440(8)$ & $\mathrm{Sm}(1)-\mathrm{O}(5)$ & $2.499(12)$ & $\mathrm{Sm}(1)-\mathrm{O}(4 \mathrm{~B})$ & $2.403(13)$ & $\mathrm{O}(6)-\mathrm{Sm}(1)-\mathrm{O}(3)$ & $73.7(2)$ \\
\hline $\mathrm{O}(4 \mathrm{~B})^{\# 1}-\mathrm{Sm}(1)-\mathrm{O}(4 \mathrm{~B})$ & $87.0(6)$ & $\mathrm{O}(6)^{\# 1}-\mathrm{Sm}(1)-\mathrm{O}(5)$ & $64.6(4)$ & $\mathrm{O}(5)-\mathrm{Sm}(1)-\mathrm{O}(3)^{\# 1}$ & $83.0(4)$ & $\mathrm{O}(4 \mathrm{~B})-\mathrm{Sm}(1)-\mathrm{O}(5)$ & $76.9(5)$ \\
\hline $\mathrm{O}(4 \mathrm{~B})^{\# 1}-\mathrm{Sm}(1)-\mathrm{O}(6)^{\# 1}$ & $85.3(4)$ & $\mathrm{O}(4 \mathrm{~B})-\mathrm{Sm}(1)-\mathrm{O}(4 \mathrm{~A})^{\# 1}$ & $76.0(4)$ & $\mathrm{O}(6)-\mathrm{Sm}(1)-\mathrm{O}(5)$ & $87.2(4)$ & $\mathrm{O}(1)-\mathrm{Sm}(1)-\mathrm{O}(2)^{\# 2}$ & $66.0(3)$ \\
\hline $\mathrm{O}(4 \mathrm{~B})-\mathrm{Sm}(1)-\mathrm{O}(6)^{\# 1}$ & $132.0(4)$ & $\mathrm{O}(5)^{\# 1}-\mathrm{Sm}(1)-\mathrm{O}(3)^{\# 1}$ & $122.7(4)$ & $\mathrm{O}(5)-\mathrm{Sm}(1)-\mathrm{O}(5)^{\# 1}$ & $40.9(8)$ & $\mathrm{O}(6)-\mathrm{Sm}(1)-\mathrm{O}(4 \mathrm{~A})$ & $112.4(4)$ \\
\hline $\mathrm{O}(6)^{\# 1}-\mathrm{Sm}(1)-\mathrm{O}(4 \mathrm{~A})$ & $152.2(4)$ & $\mathrm{O}(2)^{\# 2}-\mathrm{Sm}(1)-\mathrm{O}(5)$ & $141.0(4)$ & $\mathrm{O}(1)-\mathrm{Sm}(1)-\mathrm{O}(3)$ & $86.0(2)$ & $\mathrm{O}(1)-\mathrm{Sm}(1)-\mathrm{O}(4 \mathrm{~A})$ & $129.6(3)$ \\
\hline $\mathrm{O}(2)^{\# 2}-\mathrm{Sm}(1)-\mathrm{O}(4 \mathrm{~A})$ & 72.3(4) & $\mathrm{O}(4 \mathrm{~B})-\mathrm{Sm}(1)-\mathrm{O}(3)^{\# 1}$ & $130.3(4)$ & $\mathrm{O}(2)^{\# 2}-\mathrm{Sm}(1)-\mathrm{O}(3)$ & $71.92(18)$ & $\mathrm{O}(4 \mathrm{~B})-\mathrm{Sm}(1)-\mathrm{O}(1)$ & $134.6(3)$ \\
\hline $\mathrm{O}(5)^{\# 1}-\mathrm{Sm}(1)-\mathrm{O}(4 \mathrm{~A})$ & $68.8(5)$ & $\mathrm{O}(4 \mathrm{~B})-\mathrm{Sm}(1)-\mathrm{O}(5)^{\# 1}$ & $45.0(5)$ & $\mathrm{O}(5)-\mathrm{Sm}(1)-\mathrm{O}(3)$ & $122.7(4)$ & $\mathrm{O}(5)-\mathrm{Sm}(1)-\mathrm{O}(4 \mathrm{~A})$ & $87.8(5)$ \\
\hline $\mathrm{O}(4 \mathrm{~A})^{\# 1}-\mathrm{Sm}(1)-\mathrm{O}(4 \mathrm{~A})$ & $55.1(6)$ & $\mathrm{O}(4 \mathrm{~B})-\mathrm{Sm}(1)-\mathrm{O}(3)$ & $48.7(4)$ & $\mathrm{O}(5)^{\# 1}-\mathrm{Sm}(1)-\mathrm{O}(3)$ & $83.0(4)$ & $\mathrm{O}(4 \mathrm{~B})-\mathrm{Sm}(1)-\mathrm{O}(6)$ & $85.3(4)$ \\
\hline $\mathrm{O}(4 \mathrm{~B})^{\# 1}-\mathrm{Sm}(1)-\mathrm{O}(4 \mathrm{~A})$ & $76.0(4)$ & $\mathrm{O}(6)-\mathrm{Sm}(1)-\mathrm{O}(5)^{\# 1}$ & $64.6(4)$ & $\mathrm{O}(3)^{\# 1}-\mathrm{Sm}(1)-\mathrm{O}(3)$ & $143.1(4)$ & $\mathrm{O}(6)^{\# 1}-\mathrm{Sm}(1)-\mathrm{O}(6)$ & $66.0(3)$ \\
\hline $\mathrm{O}(4 \mathrm{~B})-\mathrm{Sm}(1)-\mathrm{O}(2)^{\# 2}$ & $96.9(4)$ & $\mathrm{O}(6)-\mathrm{Sm}(1)-\mathrm{O}(3)^{\# 1}$ & $138.8(3)$ & & & $\mathrm{O}(3)-\mathrm{Sm}(1)-\mathrm{O}(4 \mathrm{~A})$ & $54.3(3)$ \\
\hline $\mathrm{O}(3)^{\# 1}-\mathrm{Sm}(1)-\mathrm{O}(4 \mathrm{~A})$ & $107.0(4)$ & $\mathrm{O}(6)-\mathrm{Sm}(1)-\mathrm{O}(4 \mathrm{~A})^{\# 1}$ & $152.2(4)$ & $\mathrm{O}(1)-\mathrm{Sm}(1)-\mathrm{O}(3)^{\# 1}$ & $86.0(2)$ & $\mathrm{O}(6)-\mathrm{Sm}(1)-\mathrm{O}(1)$ & $78.0(3)$ \\
\hline $\mathrm{O}(6)-\mathrm{Sm}(1)-\mathrm{O}(2)^{\# 2}$ & $131.1(2)$ & & & & & & \\
\hline Symmetry codes: \#1 & 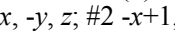 & & & & & & \\
\hline & & & & & & & \\
\hline $\operatorname{Dy}(1)-O(6 \mathrm{~A})$ & $2.368(8)$ & $\operatorname{Dy}(1)-O(4)^{\# 2}$ & $2.391(6)$ & Dy(1)-O(6B) & $2.550(9)$ & Dy(1)-O(5) & $2.463(5)$ \\
\hline Dy(1)-O(2) & $2.391(5)$ & Dy(1)-O(3) & $2.449(6)$ & Dy(1)-O(7) & $2.455(9)$ & $\mathrm{O}(2)-\mathrm{Dy}(1)-\mathrm{O}(4)^{\# 2}$ & $78.17(19)$ \\
\hline $\mathrm{O}(6 \mathrm{~A})-\mathrm{Dy}(1)-\mathrm{O}(2)^{\# 1}$ & $131.8(3)$ & $\mathrm{O}(6 \mathrm{~A})-\mathrm{Dy}(1)-\mathrm{O}(6 \mathrm{~B})$ & $27.5(3)$ & $\mathrm{O}(3)-\mathrm{Dy}(1)-\mathrm{O}(5)$ & $71.99(12)$ & $\mathrm{O}(2)^{\# 1}-\mathrm{Dy}(1)-\mathrm{O}(2)$ & $67.2(2)$ \\
\hline $\mathrm{O}(6 \mathrm{~A})^{\# 1}-\mathrm{Dy}(1)-\mathrm{O}(7)$ & $75.2(3)$ & $\mathrm{O}(6 \mathrm{~A})^{\# 1}-\mathrm{Dy}(1)-\mathrm{O}(6 \mathrm{~A})$ & $83.7(4)$ & $\mathrm{O}(7)^{\# 1}-\mathrm{Dy}(1)-\mathrm{O}(5)$ & $121.7(3)$ & $\mathrm{O}(2)-\mathrm{Dy}(1)-\mathrm{O}(7)$ & $64.3(3)$ \\
\hline $\mathrm{O}(6 \mathrm{~A})-\mathrm{Dy}(1)-\mathrm{O}(4)^{\# 2}$ & $136.2(2)$ & $\mathrm{O}(5)^{\# 1}-\mathrm{Dy}(1)-\mathrm{O}(6 \mathrm{~B})$ & $107.1(2)$ & $\mathrm{O}(7)-\mathrm{Dy}(1)-\mathrm{O}(5)$ & $83.7(3)$ & $\mathrm{O}(4)^{\# 2}-\mathrm{Dy}(1)-\mathrm{O}(5)$ & $86.35(14)$ \\
\hline $\mathrm{O}(6 \mathrm{~A})-\mathrm{Dy}(1)-\mathrm{O}(2)$ & $85.9(3)$ & $\mathrm{O}(7)^{\# 1}-\mathrm{Dy}(1)-\mathrm{O}(6 \mathrm{~B})$ & $87.4(3)$ & $\mathrm{O}(6 \mathrm{~A})-\mathrm{Dy}(1)-\mathrm{O}(5)$ & $49.9(2)$ & $\mathrm{O}(6 \mathrm{~A})-\mathrm{Dy}(1)-\mathrm{O}(7)$ & $45.4(3)$ \\
\hline $\mathrm{O}(5)-\mathrm{Dy}(1)-\mathrm{O}(6 \mathrm{~B})^{\# 1}$ & $107.1(2)$ & $\mathrm{O}(2)^{\# 1}-\mathrm{Dy}(1)-\mathrm{O}(6 \mathrm{~B})$ & $151.6(3)$ & $\mathrm{O}(7)^{\# 1}-\mathrm{Dy}(1)-\mathrm{O}(7)$ & $39.2(5)$ & $\mathrm{O}(2)-\mathrm{Dy}(1)-\mathrm{O}(6 \mathrm{~B})$ & $111.5(2)$ \\
\hline O(6A)-Dy(1)-O(3) & $96.4(3)$ & $\mathrm{O}(2)-\mathrm{Dy}(1)-\mathrm{O}(6 \mathrm{~B})^{\# 1}$ & $151.6(3)$ & $\mathrm{O}(2)^{\# 1}-\mathrm{Dy}(1)-\mathrm{O}(5)$ & $139.59(16)$ & $\mathrm{O}(3)-\mathrm{Dy}(1)-\mathrm{O}(7)$ & 141.1(3) \\
\hline $\mathrm{O}(7)-\mathrm{Dy}(1)-\mathrm{O}(6 \mathrm{~B})^{\# 1}$ & $87.4(3)$ & $\mathrm{O}(6 \mathrm{~A})^{\# 1}-\mathrm{Dy}(1)-\mathrm{O}(5)$ & $128.4(3)$ & $\mathrm{O}(2)-\mathrm{Dy}(1)-\mathrm{O}(5)^{\# 1}$ & $139.59(16)$ & $\mathrm{O}(3)-\mathrm{Dy}(1)-\mathrm{O}(6 \mathrm{~B})$ & $72.0(2)$ \\
\hline $\mathrm{O}(4)^{\# 2}-\mathrm{Dy}(1)-\mathrm{O}(3)$ & $66.98(19)$ & $\mathrm{O}(6 \mathrm{~B})^{\# 1}-\mathrm{Dy}(1)-\mathrm{O}(6 \mathrm{~B})$ & $55.3(4)$ & $\mathrm{O}(2)-\mathrm{Dy}(1)-\mathrm{O}(3)$ & $131.52(17)$ & $\mathrm{O}(2)-\mathrm{Dy}(1)-\mathrm{O}(5)$ & $73.21(16)$ \\
\hline $\mathrm{O}(6 \mathrm{~A})-\mathrm{Dy}(1)-\mathrm{O}(7)^{\# 1}$ & $75.2(3)$ & $\mathrm{O}(3)-\mathrm{Dy}(1)-\mathrm{O}(6 \mathrm{~B})^{\# 1}$ & $72.0(2)$ & $\mathrm{O}(3)-\mathrm{Dy}(1)-\mathrm{O}(5)^{\# 1}$ & $71.99(12)$ & $\mathrm{O}(7)-\mathrm{Dy}(1)-\mathrm{O}(6 \mathrm{~B})$ & $69.1(3)$ \\
\hline $\mathrm{O}(2)-\mathrm{Dy}(1)-\mathrm{O}(7)^{\# 1}$ & $86.4(3)$ & $\mathrm{O}(4)^{\# 2}-\mathrm{Dy}(1)-\mathrm{O}(6 \mathrm{~B})$ & $130.1(2)$ & $\mathrm{O}(7)-\mathrm{Dy}(1)-\mathrm{O}(5)^{\# 1}$ & $121.7(3)$ & $\mathrm{O}(5)-\mathrm{Dy}(1)-\mathrm{O}(6 \mathrm{~B})$ & $54.0(2)$ \\
\hline
\end{tabular}




\begin{tabular}{|c|c|c|c|c|c|c|c|}
\hline $\mathrm{O}(3)-\mathrm{Dy}(1)-\mathrm{O}(7)^{\# 1}$ & $141.1(3)$ & $\mathrm{O}(6 \mathrm{~A})^{\# 1}-\mathrm{Dy}(1)-\mathrm{O}(6 \mathrm{~B})$ & $74.4(3)$ & $\mathrm{O}(5)-\mathrm{Dy}(1)-\mathrm{O}(5)^{\# 1}$ & $143.2(2)$ & $\mathrm{O}(4)^{\# 2}-\mathrm{Dy}(1)-\mathrm{O}(7)$ & $142.5(3)$ \\
\hline \multicolumn{8}{|c|}{$\begin{array}{l}\text { Symmetry codes: } \# 1 x,-y+1, z ; \# 2-x+1,-y+1,-z+1 \text {. } \\
\text { Complex } 6\end{array}$} \\
\hline $\mathrm{Eu}(1)-\mathrm{O}(8)$ & $2.383(14)$ & $\mathrm{Eu}(1)-\mathrm{O}(1)$ & $2.463(13)$ & $\mathrm{Eu}(1)-\mathrm{O}(2)^{\# 3}$ & $2.473(9)$ & $\mathrm{Eu}(1)-\mathrm{O}(9)$ & $2.593(16)$ \\
\hline $\mathrm{Eu}(1)-\mathrm{O}(5)$ & $2.429(8)$ & $\mathrm{Eu}(1)-\mathrm{O}(10)$ & $2.463(16)$ & $\mathrm{Eu}(1)-\mathrm{O}(3)$ & $2.516(8)$ & $\mathrm{O}(8)-\mathrm{Eu}(1)-\mathrm{O}(5)^{\# 5}$ & $131.6(4)$ \\
\hline $\mathrm{O}(8)-\mathrm{Eu}(1)-\mathrm{O}(5)$ & $84.9(5)$ & $\mathrm{O}(5)-\mathrm{Eu}(1)-\mathrm{O}(10)$ & $64.8(5)$ & $\mathrm{O}(1)-\mathrm{Eu}(1)-\mathrm{O}(3)$ & $86.6(3)$ & $\mathrm{O}(8)-\mathrm{Eu}(1)-\mathrm{O}(9)^{\# 5}$ & $76.6(5)$ \\
\hline $\mathrm{O}(5)^{\# 5}-\mathrm{Eu}(1)-\mathrm{O}(5)$ & $66.6(4)$ & $\mathrm{O}(1)-\mathrm{Eu}(1)-\mathrm{O}(10)$ & $142.3(5)$ & $\mathrm{O}(10)^{\# 5}-\mathrm{Eu}(1)-\mathrm{O}(3)$ & $121.3(5)$ & $\mathrm{O}(5)-\mathrm{Eu}(1)-\mathrm{O}(9)^{\# 5}$ & $153.0(4)$ \\
\hline $\mathrm{O}(8)-\mathrm{Eu}(1)-\mathrm{O}(1)$ & $135.1(4)$ & $\mathrm{O}(8)-\mathrm{Eu}(1)-\mathrm{O}(2)^{\# 3}$ & $97.8(4)$ & $\mathrm{O}(10)-\mathrm{Eu}(1)-\mathrm{O}(3)$ & $83.6(5)$ & $\mathrm{O}(3)-\mathrm{Eu}(1)-\mathrm{O}(9)^{\# 5}$ & $107.0(4)$ \\
\hline $\mathrm{O}(5)-\mathrm{Eu}(1)-\mathrm{O}(1)$ & $77.5(3)$ & $\mathrm{O}(5)-\mathrm{Eu}(1)-\mathrm{O}(2)^{\# 3}$ & $130.6(3)$ & $\mathrm{O}(2)^{\# 3}-\mathrm{Eu}(1)-\mathrm{O}(3)$ & $72.2(2)$ & $\mathrm{O}(5)-\mathrm{Eu}(1)-\mathrm{O}(9)$ & $112.1(4)$ \\
\hline $\mathrm{O}(8)-\mathrm{Eu}(1)-\mathrm{O}(10)^{\# 5}$ & $75.6(6)$ & $\mathrm{O}(1)-\mathrm{Eu}(1)-\mathrm{O}(2)^{\# 3}$ & $66.1(3)$ & $\mathrm{O}(8)-\mathrm{Eu}(1)-\mathrm{O}(3)^{\# 5}$ & $129.9(5)$ & $\mathrm{O}(1)-\mathrm{Eu}(1)-\mathrm{O}(9)$ & $129.3(4)$ \\
\hline $\mathrm{O}(3)-\mathrm{Eu}(1)-\mathrm{O}(9)$ & $53.4(4)$ & $\mathrm{O}(10)-\mathrm{Eu}(1)-\mathrm{O}(2)^{\# 3}$ & $142.2(4)$ & $\mathrm{O}(5)-\mathrm{Eu}(1)-\mathrm{O}(3)^{\# 5}$ & $139.3(3)$ & $\mathrm{O}(10)^{\# 5}-\mathrm{Eu}(1)-\mathrm{O}(9)$ & $88.3(5)$ \\
\hline $\mathrm{O}(8)^{\# 5}-\mathrm{Eu}(1)-\mathrm{O}(10)$ & $75.6(6)$ & $\mathrm{O}(8)^{\# 5}-\mathrm{Eu}(1)-\mathrm{O}(3)$ & $129.9(5)$ & $\mathrm{O}(10)-\mathrm{Eu}(1)-\mathrm{O}(3)^{\# 5}$ & $121.3(5)$ & $\mathrm{O}(10)-\mathrm{Eu}(1)-\mathrm{O}(9)$ & $70.0(5)$ \\
\hline $\mathrm{O}(8)-\mathrm{Eu}(1)-\mathrm{O}(10)$ & $45.3(5)$ & $\mathrm{O}(8)-\mathrm{Eu}(1)-\mathrm{O}(3)$ & $48.7(4)$ & $\mathrm{O}(3)-\mathrm{Eu}(1)-\mathrm{O}(3)^{\# 5}$ & $143.4(4)$ & $\mathrm{O}\left(2^{* \# 3}-\mathrm{Eu}(1)-\mathrm{O}(9)\right.$ & $72.2(4)$ \\
\hline $\mathrm{O}(5)^{\# 5}-\mathrm{Eu}(1)-\mathrm{O}(10)$ & $86.5(5)$ & $\mathrm{O}(5)-\mathrm{Eu}(1)-\mathrm{O}(3)$ & $73.4(3)$ & & & & \\
\hline \multicolumn{8}{|c|}{$\begin{array}{l}\text { Symmetry codes: } \# 3-x+1,-y,-z+1 ; \# 5 x,-y, z \text {. } \\
\text { Complex } 7\end{array}$} \\
\hline $\mathrm{O}(7)-\mathrm{Eu}(1)$ & $2.302(3)$ & $\mathrm{Eu}(1)-\mathrm{O}(3)$ & $2.403(3)$ & $\mathrm{Eu}(1)-\mathrm{O}(4)$ & $2.434(3)$ & $\mathrm{Eu}(1)-\mathrm{O}(6)$ & $2.438(2)$ \\
\hline $\mathrm{Eu}(1)-\mathrm{O}(2)$ & $2.268(3)$ & $\mathrm{Eu}(1)-\mathrm{N}(3)$ & $2.423(3)$ & $\mathrm{Eu}(1)-\mathrm{N}(1)$ & $2.436(3)$ & $\mathrm{Eu}(1)-\mathrm{O}(5)$ & $2.454(2)$ \\
\hline $\mathrm{O}(2)-\mathrm{Eu}(1)-\mathrm{O}(7)$ & $81.12(10)$ & $\mathrm{O}(7)-\mathrm{Eu}(1)-\mathrm{O}(4)$ & $145.23(10)$ & $\mathrm{O}(4)-\mathrm{Eu}(1)-\mathrm{N}(1)$ & $96.53(12)$ & $\mathrm{O}(2)-\mathrm{Eu}(1)-\mathrm{O}(5)$ & $144.04(9)$ \\
\hline $\mathrm{O}(2)-\mathrm{Eu}(1)-\mathrm{O}(3)$ & $77.10(10)$ & $\mathrm{O}(3)-\mathrm{Eu}(1)-\mathrm{O}(4)$ & $56.86(10)$ & $\mathrm{O}(2)-\mathrm{Eu}(1)-\mathrm{O}(6)$ & $145.01(9)$ & $\mathrm{O}(7)-\mathrm{Eu}(1)-\mathrm{O}(5)$ & $75.31(10)$ \\
\hline $\mathrm{O}(7)-\mathrm{Eu}(1)-\mathrm{O}(3)$ & $157.54(11)$ & $\mathrm{N}(3)-\mathrm{Eu}(1)-\mathrm{O}(4)$ & $91.53(12)$ & $\mathrm{O}(7)-\mathrm{Eu}(1)-\mathrm{O}(6)$ & $76.68(10)$ & $\mathrm{O}(3)-\mathrm{Eu}(1)-\mathrm{N}(1)$ & $78.62(11)$ \\
\hline $\mathrm{O}(2)-\mathrm{Eu}(1)-\mathrm{N}(3)$ & $78.38(11)$ & $\mathrm{O}(2)-\mathrm{Eu}(1)-\mathrm{N}(1)$ & $78.03(11)$ & $\mathrm{O}(3)-\mathrm{Eu}(1)-\mathrm{O}(6)$ & $119.45(9)$ & $\mathrm{N}(3)-\mathrm{Eu}(1)-\mathrm{O}(5)$ & $77.46(9)$ \\
\hline $\mathrm{O}(7)-\mathrm{Eu}(1)-\mathrm{N}(3)$ & $95.83(12)$ & $\mathrm{O}(3)-\mathrm{Eu}(1)-\mathrm{O}(5)$ & $126.45(10)$ & $\mathrm{N}(3)-\mathrm{Eu}(1)-\mathrm{O}(6)$ & $130.22(10)$ & $\mathrm{O}(4)-\mathrm{Eu}(1)-\mathrm{O}(5)$ & $73.25(9)$ \\
\hline $\mathrm{O}(3)-\mathrm{Eu}(1)-\mathrm{N}(3)$ & $85.15(11)$ & $\mathrm{N}(1)-\mathrm{Eu}(1)-\mathrm{O}(5)$ & $128.74(10)$ & $\mathrm{O}(4)-\mathrm{Eu}(1)-\mathrm{O}(6)$ & $72.73(10)$ & $\mathrm{O}(7)-\mathrm{Eu}(1)-\mathrm{N}(1)$ & $91.65(13)$ \\
\hline $\mathrm{O}(2)-\mathrm{Eu}(1)-\mathrm{O}(4)$ & $133.63(10)$ & N(3)-Eu(1)-N(1) & $153.80(11)$ & $\mathrm{N}(1)-\mathrm{Eu}(1)-\mathrm{O}(6)$ & $75.95(10)$ & $\mathrm{O}(6)-\mathrm{Eu}(1)-\mathrm{O}(5)$ & $52.89(8)$ \\
\hline \multicolumn{8}{|l|}{ Complex 8} \\
\hline $\mathrm{N}(2)-\mathrm{Tb}(1)$ & $2.456(4)$ & $\mathrm{O}(1)-\mathrm{Tb}(1)$ & $2.286(3)$ & $\mathrm{O}(4)-\mathrm{Tb}(1)$ & $2.435(4)$ & $\mathrm{O}(6)-\mathrm{Tb}(1)$ & $2.461(3)$ \\
\hline $\mathrm{N}(3)-\mathrm{Tb}(1)$ & $2.446(4)$ & $\mathrm{O}(3)-\mathrm{Tb}(1)$ & $2.454(4)$ & $\mathrm{O}(5)-\mathrm{Tb}(1)$ & $2.469(3)$ & $\mathrm{O}(7)-\mathrm{Tb}(1)$ & $2.334(4)$ \\
\hline $\mathrm{O}(1)-\mathrm{Tb}(1)-\mathrm{O}(7)$ & 81.64(13) & $\mathrm{O}(7)-\mathrm{Tb}(1)-\mathrm{O}(3)$ & $145.32(14)$ & $\mathrm{O}(3)-\mathrm{Tb}(1)-\mathrm{N}(2)$ & $96.65(17)$ & $\mathrm{O}(7)-\mathrm{Tb}(1)-\mathrm{N}(2)$ & $91.48(16)$ \\
\hline $\mathrm{O}(1)-\mathrm{Tb}(1)-\mathrm{O}(4)$ & $76.87(14)$ & $\mathrm{O}(4)-\mathrm{Tb}(1)-\mathrm{O}(3)$ & $56.48(15)$ & $\mathrm{O}(1)-\mathrm{Tb}(1)-\mathrm{O}(6)$ & $145.39(12)$ & $\mathrm{O}(7)-\mathrm{Tb}(1)-\mathrm{O}(5)$ & $75.57(13)$ \\
\hline $\mathrm{O}(7)-\mathrm{Tb}(1)-\mathrm{O}(4)$ & $157.81(15)$ & $\mathrm{N}(3)-\mathrm{Tb}(1)-\mathrm{O}(3)$ & $92.10(17)$ & $\mathrm{O}(7)-\mathrm{Tb}(1)-\mathrm{O}(6)$ & $76.52(13)$ & $\mathrm{O}(4)-\mathrm{Tb}(1)-\mathrm{N}(2)$ & $78.74(16)$ \\
\hline $\mathrm{O}(1)-\mathrm{Tb}(1)-\mathrm{N}(3)$ & $78.11(14)$ & $\mathrm{O}(4)-\mathrm{Tb}(1)-\mathrm{O}(5)$ & $125.97(14)$ & $\mathrm{O}(4)-\mathrm{Tb}(1)-\mathrm{O}(6)$ & $119.40(14)$ & $\mathrm{N}(3)-\mathrm{Tb}(1)-\mathrm{O}(5)$ & $77.43(12)$ \\
\hline $\mathrm{O}(7)-\mathrm{Tb}(1)-\mathrm{N}(3)$ & $95.31(16)$ & $\mathrm{N}(2)-\mathrm{Tb}(1)-\mathrm{O}(5)$ & $128.84(12)$ & $\mathrm{N}(3)-\mathrm{Tb}(1)-\mathrm{O}(6)$ & $130.00(12)$ & $\mathrm{O}(3)-\mathrm{Tb}(1)-\mathrm{O}(5)$ & $73.11(12)$ \\
\hline $\mathrm{O}(4)-\mathrm{Tb}(1)-\mathrm{N}(3)$ & $85.68(16)$ & $\mathrm{O}(1)-\mathrm{Tb}(1)-\mathrm{O}(5)$ & $144.59(12)$ & $\mathrm{O}(3)-\mathrm{Tb}(1)-\mathrm{O}(6)$ & $72.85(15)$ & $\mathrm{O}(1)-\mathrm{Tb}(1)-\mathrm{N}(2)$ & $77.81(13)$ \\
\hline $\mathrm{O}(1)-\mathrm{Tb}(1)-\mathrm{O}(3)$ & $133.02(13)$ & $\mathrm{N}(3)-\mathrm{Tb}(1)-\mathrm{N}(2)$ & $153.72(14)$ & $\mathrm{N}(2)-\mathrm{Tb}(1)-\mathrm{O}(6)$ & $76.27(13)$ & $\mathrm{O}(6)-\mathrm{Tb}(1)-\mathrm{O}(5)$ & $52.65(10)$ \\
\hline
\end{tabular}

